\title{
Advanced fluid models for resonant online oil condition monitoring systems
}

\author{
Neue Fluidmodelle für resonant arbeitende Ölzustandssensoren
}

https://doi.org/10.1515/teme-2021-0108

Received September 30, 2021; accepted October 1, 2021
Schlagwörter: Instandhaltung, Viskosität, Dichte, Temperaturkoeffizient, Sensorik.

\section{Introduction}

This article extends the two conference contributions [1, 2], which showed a convenient new representation of the viscosity-temperature dependence of fluids and a model relating resonance characteristics of resonators immersed in fluids. Further insights and results are presented alongside a detailed discussion of the role of lubricant viscosity in maintenance.

To reduce maintenance costs as well as the risk of unplanned downtimes, the industry gradually adopts online condition monitoring (OCM) methods combined with predictive or proactive maintenance approaches. Enabled by the increasing level of automation, plenty of data can be made available to maintenance personnel and condition monitoring algorithms, and sophisticated methods can be implemented to assist in the planning of maintenance actions. With the implementation of such data-based decision methods, the reliability and precision of the collected data have a significant impact on the effectiveness of the maintenance actions triggered. Furthermore, the sooner a problem can be identified, the easier and cheaper the appropriate maintenance action will be. So in many cases, the benefit of a sensor increases over proportionately with its accuracy and long-term stability.

As is outlined in Gulati and Smith [3], reliabilitycentered maintenance (RCM) provides a structured framework for analyzing the function and potential failures of assets. RCM utilizes various maintenance optimizing strategies including condition-based (CBM) and preventive (PM) maintenance, but also run-to-failure (RTF), for instance. In RMC, the potential-failure, or P-F, curve shown in Fig. 1, is considered one the most important tools. It shows the asset condition over time. The most crucial part of the chart is the so-called P-F interval (also known as the lead time to failure), which represents the time between the detection of a potential failure and functional failure. The length of the interval and therefore the 


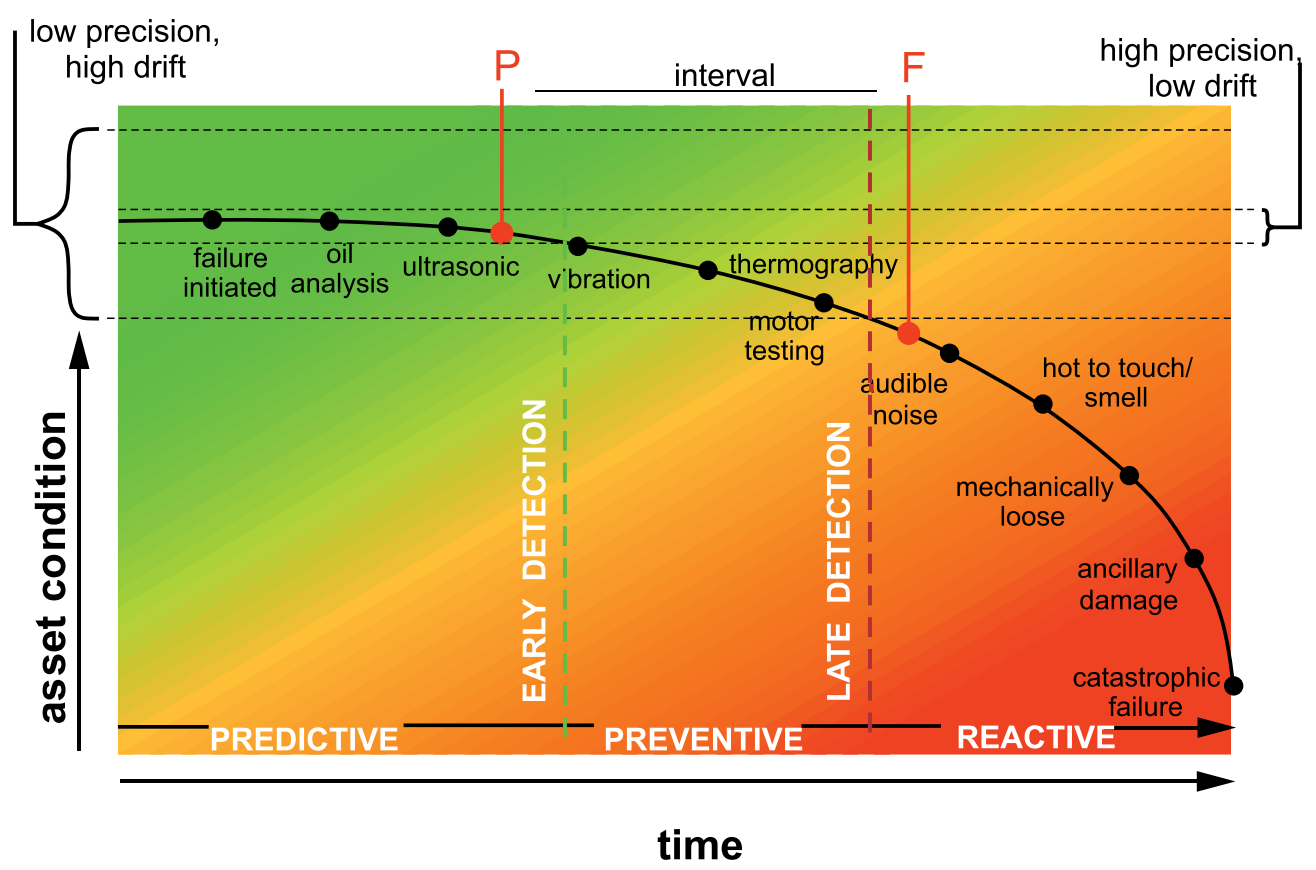

Figure 1: The prominent P-F curve is an important tool in maintenance for developing strategies in the framework of reliability-centered maintenance (RCM). Oil analysis is suitable for early detection of potential failure states (P) and thus enables the establishment of an economic maintenance plan in order to avoid functional failure (F).

time to react is determined by the technology used to detect a failure and the frequency of inspection. According to [4] and [5], oil analysis is capable to detect a potential failure state early. Mobley [6, p. 203] notes, that traditional sampled oil analysis has become an important aid to preventive PM. The typically performed tests include viscosity, contamination, fuel dilution, solids content, fuel soot, oxidation, nitration, total acid number (TAN), total base number (TBN), particle count, and spectrographic analysis. Among the analyzed properties viscosity is denoted as one of the most important properties of lubricating oils. $[3$, p. 223] defines the role of viscosity in more detail as follows:

“...It is also often referred to as the structural strength of liquid. Viscosity is critical to oil film control and is a key indicator of condition related to the oil and the machine."

Figure 2 compares aspects of online sensors for oil analysis and traditional laboratory analysis. Generally, online sensors cannot be considered miniaturized laboratory instruments, but instead, face much harsher conditions than in a controlled laboratory setting. These include, that the online sensor systems cannot be cleaned before each measurement, cannot be calibrated each day, have to withstand temperature cycles and sometimes high hydraulic pressures. Online sensors however can only capture a limited amount of oil properties. To meet the prize expecta- tions of customers, it is required to focus on the measurement of few, but most meaningful quantities while providing a high ruggedness of the system at the same time.

\subsection{Monitoring system}

The fluidFOX shown in Fig. 3a is a novel fully automated online condition monitoring system for hydraulic fluids and lubricating oils [7]. By using a vibrating sensor element, viscosity and density can be measured simultaneously. As there are no rotating parts, no bearings or shaft seals are required. This enhances robustness and measurement accuracy particularly at low viscosities ${ }^{1}$ and allows pressure-resistant designs that can withstand high hydraulic pressures [8]. The low volume measurement cell allows the desired temperature setpoints to be reached quickly [9].

The temperature-controlled measurement cell within the system houses the vibrating quartz tuning fork sensor (QTF), a Pt100 temperature sensor, and a capacitive relative-humidity sensor as shown in Fig. 3. From the fluidinduced resonance changes of the QTF, the viscosity and

1 I. e., also gases can be measured, but which is not further discussed here. 


\section{online} sensor systems

+ very fast

+ online, i.e. 14.0, IIOT ready

+ limited measurement range required

- limited number of parameters

\section{oil condition monitoring}

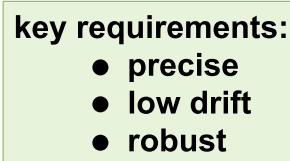

\section{laboratory analysis} ("bottle sampling")

+ very accurate

+ standardized measurements

+ many parameters

- expensive

- slow

- prone to handling errors

\section{design challenges for online sensors:}

- for rough environmental conditions (pressure, temperature, vibration)

- no cleaning before each measurement possible no ongoing check / calibration possible

- customer expectation: much cheaper than a laboratory instrument!

Figure 2: Oil condition monitoring using online sensors versus laboratory analysis. Online sensors face several design challenges.
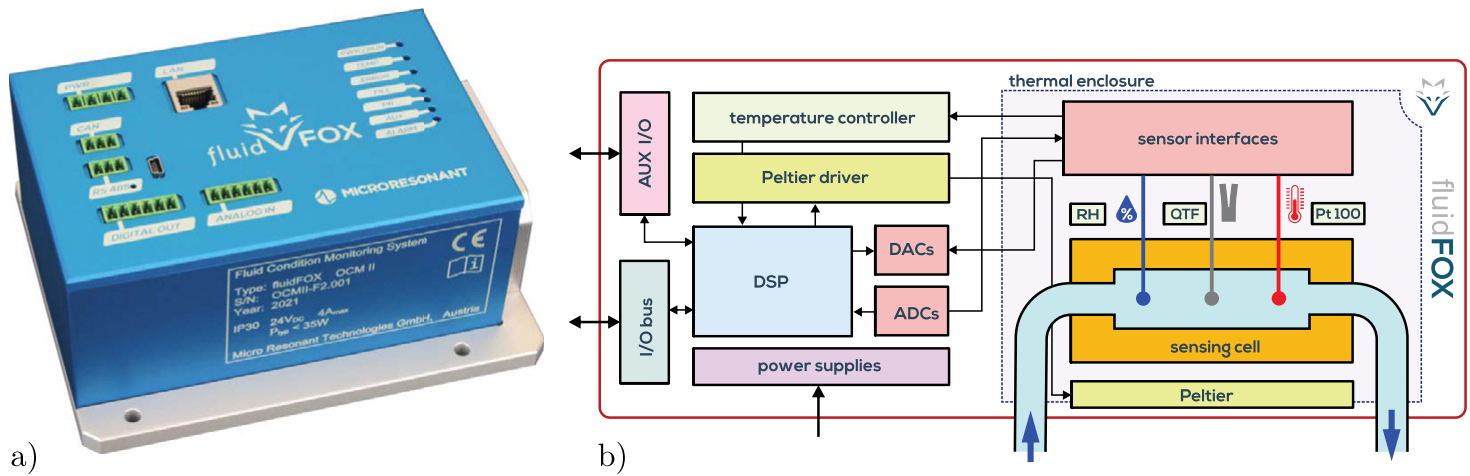

Figure 3: a) Online condition monitoring system "fluidFOX” and its internal components shown in b).

density of the fluid are determined [10, 11]. As viscosity shows significant temperature dependence, it must be measured at well-defined temperatures. Therefore, a precise thermoelectric temperature controller is implemented which can be used to cycle temperature and to determine additional characteristic fluid parameters. These comprise the temperature coefficient of the density but also various viscosity-temperature indices such as the VTC, the VI, or the m-value [12]. Although not discussed in this publication, the system also provides the electrical fluid parameters and the relative humidity, which, if measured over temperature, yields a multi-parameter characterization of the fluid under test [8].

\section{Theory of operation}

The resonance characteristics of vibrating structure immersed in liquid change due to inertial and dissipative ef- fects, which are associated with the fluid density and the viscosity. From changes of resonance frequency and quality factor, fluid viscosity and density can be calculated, given a fluid model is established. Fig. 4 a shows the quartz tuning fork (QTF) sensor and Fig. 4b the resonance curves for relevant technical fluids (b). The resonance frequency and the $Q$-factor shown in Fig. 4 c were estimated from these spectral data. The fluid model relates these quantities to density and kinematic viscosity $(v)$. As also the density is determined, the dynamic viscosity $(\eta)$ can be obtained by $\eta=v \rho_{\mathrm{f}}$.

Figure 5a shows an intuitive illustration of the measurement principle, that although it may appear different in nature, captures the main features of the fluid-loaded vibrating tuning fork sensor. A heavy sphere of radius $R$ and mass $m$ attached to a rigidly mounted spring with constant $k$ and some (unwanted) damping $d$ vibrates with angular frequency $\omega$ in a fluid of density $\rho_{\mathrm{f}}$ and dynamic viscosity $\eta$. As the sphere moves up and down, fluid mass is pe- 


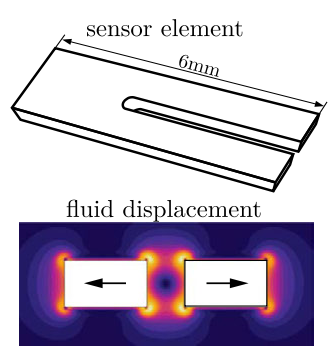

a)

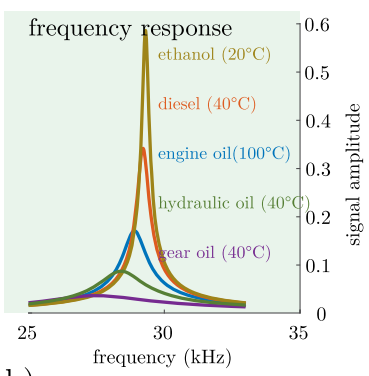

b)

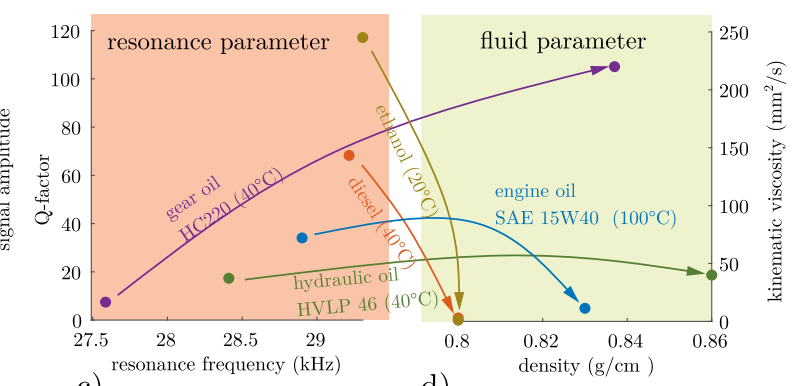

c)

d)

Figure 4: a) Actual QTF with fluid displacement profile. b) Frequency resonance of magnitude for various fluids. c) The resonance parameters are estimated from the frequency responses. $d$ ) The estimated fluid density and viscosity.

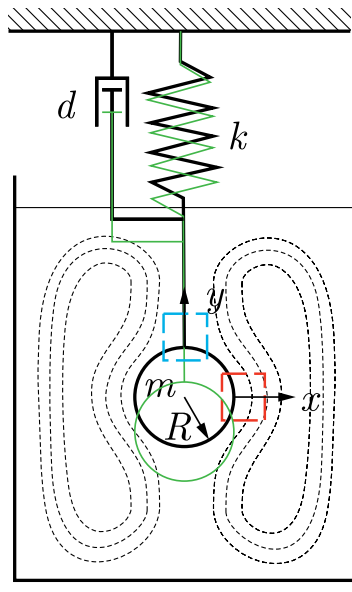

a)

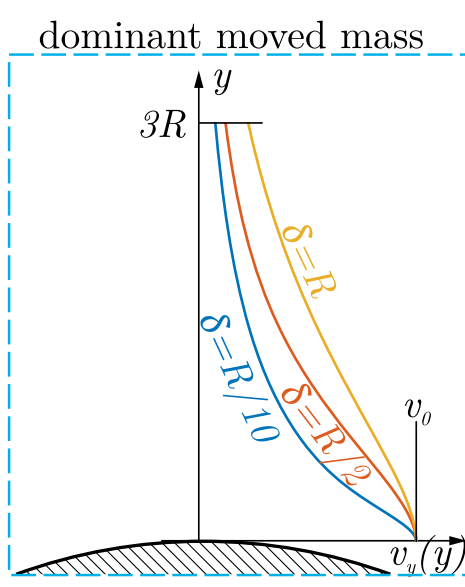

b) dominant shear regime

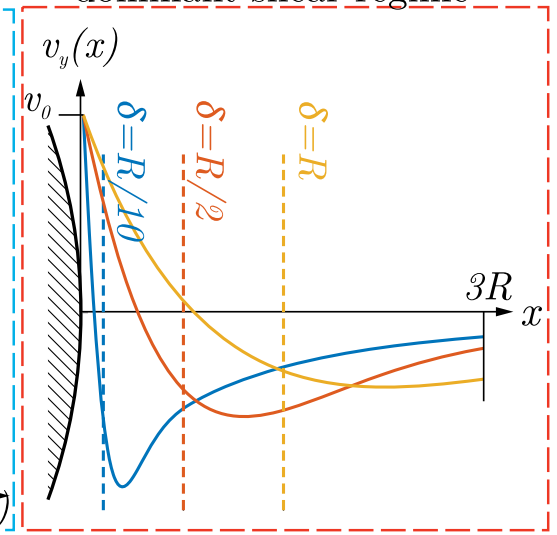

c)

Figure 5: a) A vibrating sphere on a spring as an instructive example for a resonant fluid sensor. b) The velocity profile in direction of motion at the top of the sphere shows a dominant moved mass regime. $c$ ) The velocity profile perpendicular to the motion indicates a dominant shear flow regime.

riodically displaced and the oscillation is damped by the fluid viscosity $\eta$. As the fluid sticks to the surface of the sphere (i.e., the no-slip condition), a certain amount of fluid is dragged due to viscous effects too, increasing the moved mass for higher viscosities. Figure $5 b$, c show the fluid particle velocities along the $x$ and $y$ axes at the positions indicated in a). The figures show areas with dominant mass displacement (b) and shear stress (c), respectively. The characteristic dimensionless quantity relevant for the problem is the ratio $\delta / R$, where higher values correspond to higher kinematic viscosity $v$. The meaning of this quantity is discussed in more detail later. For the time being, the plots can be interpreted such that higher viscosities are associated with moderately increased mass (see b), but with strongly altered shear displacements (see c). Higher viscosities generally cause increased fluid damping, as long as the shear waves do not strongly interact with fluid container walls.
Fluid forces $F_{\mathrm{f}}$ acting on the structure damp the driven oscillation with damping $d_{\mathrm{f}}$ and cause a shift in oscillation frequency due to dragged mass $m_{\mathrm{f}}$. The equation of the spring-mass-damper system and the equation for the fluid force $F_{\mathrm{f}}$ acting on a vibrating body are therefore

$$
F(t)=m \ddot{x}(t)+d \dot{x}(t)+k x(t)+F_{\mathrm{f}}(t)
$$

with $\quad F_{\mathrm{f}}(t)=m_{\mathrm{f}} \ddot{x}(t)+d_{\mathrm{f}} \dot{x}(t)$.

The two resonance parameters characterizing the vibration are the resonance frequency $f_{\mathrm{r}}$ and the quality factor $Q$, for which the following definitions are used:

$$
f_{\mathrm{r}}=\frac{1}{2 \pi} \sqrt{\frac{k}{m+m_{\mathrm{f}}}} \text { and } Q=\frac{\sqrt{k\left(m+m_{\mathrm{f}}\right)}}{d_{f}} .
$$

According to [13], the fluid force acting on a harmonically oscillating sphere in unbounded fluid in the Fourier do- 
main (i. e., using $d(..) / d t \circ \longrightarrow j \omega \cdot(. .)$.$) is given by$

$$
\begin{aligned}
& \hat{\underline{F}}_{\mathrm{f}}(\omega)=-\omega^{2} \rho_{\mathrm{f}} \frac{4 R^{3} \pi}{3} \underbrace{\left(\frac{1}{2}+\frac{9 \delta}{4 R}-j \frac{9}{4} \frac{\delta}{R}-j \frac{9}{4}\left(\frac{\delta}{R}\right)^{2}\right)}_{\underline{\Gamma}(\delta / R)} \underline{\hat{\underline{x}}}(\omega) \\
& \text { with } \delta=\sqrt{\frac{2 \eta}{\rho_{\mathrm{f}} \omega}} .
\end{aligned}
$$

Underlined quantities are complex-valued, and hat symbols indicate complex amplitudes. The dimensionless hydrodynamic function $\underline{\Gamma}$ represents a complex-valued polynomial in $\delta / R$ of second order. Closed-form expressions for the fluid force are only available for some very simple geometries including spheres, cylinders, or blades. E. g., the expression for vibrating cylinders [14] involves a complicated dependence of $\underline{\Gamma}$ on $\delta / R$. It can be shown that a power series of the expression truncated to order of two yields the same form for resonance frequency and $Q$-factor as for the sphere, but with different coefficients. A model motivated by these findings was derived by [15] using six adjustable parameters $\left(c_{1} \ldots c_{6}\right)$

$$
\begin{aligned}
f_{\mathrm{r}} & =\left(c_{1}+c_{2} \rho_{\mathrm{f}}+c_{3} \sqrt{\rho_{\mathrm{f}} \eta / f_{\mathrm{r}}}\right)^{-1 / 2} \text { and } \\
Q & =\left(c_{4}+c_{5} \eta+c_{6} \sqrt{\rho_{\mathrm{f}} \eta f_{\mathrm{r}}}\right)^{-1} .
\end{aligned}
$$

\subsection{An extended fluid model}

Models derived using the same line or arguments have been reported by different groups $[16,17,18]$ yielding different representations with a different number of constants. Although these models have successfully been used for a host of fluid sensors including tuning fork sensors, the user must be aware of the underlying truncation that limits the measurement range for which the approximation is accurate enough. Using a higher order of approximation is the proposed solution to extend the overall measurement accuracy and the measurement range. However, extending the model in (4) is not straight-foreward in practice, as it requires adaptions of the parameter calibration process and model inversion (i. e., calculating $\eta$ and $\rho_{\mathrm{f}}$ from $f_{\mathrm{r}}$ and $Q$ ). Based on the findings in [19] the polynomial model using complex-valued coefficients $\underline{a}_{n}$ is proposed as follows:

$$
\underline{G}\left(f_{\mathrm{r}}, Q\right)=\rho_{\mathrm{f}} \sum_{n=0}^{N} \underline{a}_{n} \zeta^{n} \text { with } \zeta=\sqrt{v / f_{\mathrm{r}}}
$$

$\underline{G}$ is a complex-valued function of the measured resonance parameters $f_{\mathrm{r}}$ and $Q$, only. Motivated by dimensional analysis, it is found in [19], that this form is suitable representation for any vibrating structure in simple Newtonian fluids in rigid housing. Even for strong fluid interactions with the (rigid) container walls (see Fig. 5), the form is applicable, but the polynomial degree needed, may be larger. The polynomial coefficients $\underline{a}_{n}$ are adjusted for an actual sensor setup by reference measurements using test fluids that cover the desired measurement range. Solving (5) is numerically straight-forward for any polynomial degree $N$. Finding the correct root $\zeta$ among the $N$ possible roots, poses no difficulty for a well designed sensor which features a one-to-one relation between $\left\{f_{\mathrm{r}}, Q\right\}$ and $\left\{\rho_{\mathrm{f}}, \nu\right\}$ in the desired measurement range. This corresponds to a monotonic polynomial in (5) with only one real root in the calibration range. It is emphasized that the polynomial can only be applied in the calibration range (i. e., by interpolation). According to Descartes' rule of signs, there may be spurious real-valued roots outside the calibration range. Convergence to spurious roots is difficult to avoid by using the common Newton method, for instance. Despite their slower convergence rate, it is suggested to use bracketing methods for which convergence to the correct root can be assured. When using root finding methods that yield all roots at once, such as Aberth's method [20, 21], the correct root is the (most) real-valued root within the calibration range. A comparison to the state-of-the art models reported in $[15,18,17]$ is shown in Fig. 6 . The alternative models can be transformed into the model in (5) for $N=2$. The here reported model is therefore a higher order extension to the previous models. By using the new model, the parameter estimation $\left(\underline{a}_{n}\right)$ and the calculation of fluid parameters from measured resonance parameters remains straight-forward for any order $N$, while extension of the alternative models in a similar fashion is not obvious. The benefit of the new model is the flexibility to adjust the degree of approximation to any higher order, i. e., to enhance the measurement range and accuracy of interpolation.

\section{Temperature models}

First empirical viscosity-temperature models were already compared in [22] in 1897, where the earliest mentioned model dates back to 1843 and was introduced by Poiseuille [23] reading

$$
\eta=\frac{C}{1+a T+b T^{2}} .
$$

This and other early models could only be applied in a small temperature range. The situation was improved with the discovery the exponential temperature dependence of physical and chemical processes by Arrhenius [24] in 1889 


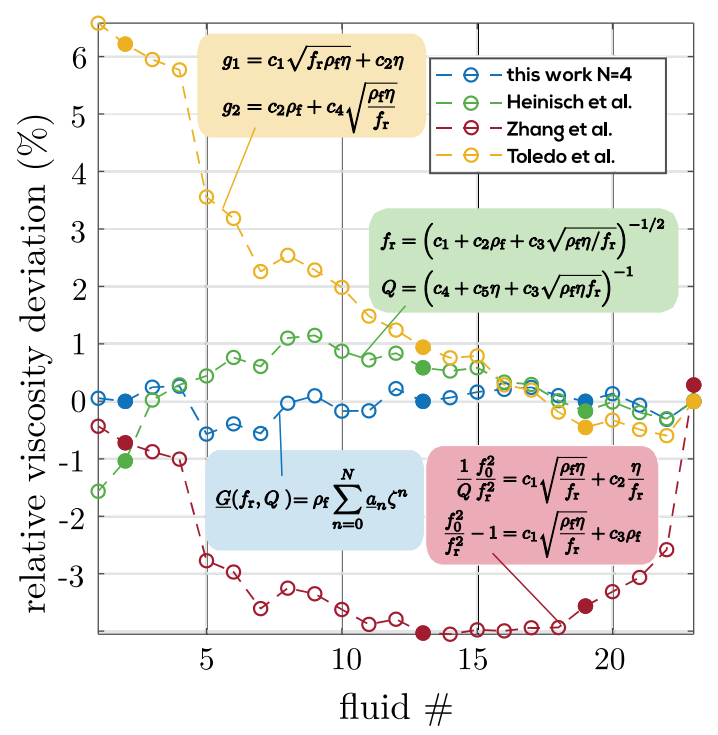

Figure 6: The parameters for the model in (5) and three models from $[15,18,17]$ were adjusted using the certified test fluids marked with $a *$ symbol in the table on the right. The deviations between estimated viscosities are compared in the graph for all fluids.

which resulted in a more accurate two-parameter model, where $T$ denotes the absolute temperature

$$
\eta=A \exp \frac{B}{T}
$$

The myriad of subsequently introduced models are in many cases empirical extensions of the Arrhnenius model using more parameters for better agreement, use other exponential bases, or are simply different representations of another model (see e.g. [25]). Particularly with condition monitoring, however, it is also necessary that the parameters have a clear physical meaning. As the number of model parameters increases, it becomes more and more difficult to interpret the individual parameters. It is therefore important to use the model with the fewest parameters while ensuring accuracy for the examined fluid class and lowest covariance of parameters. The latter aspect deserves particular attention as inevitable measurement errors on viscosity result in noise on the estimated model parameters. For example, the Arrhenius model, extended by parameter $C$

$$
\eta=A \exp (B / T+C)
$$

has infinitely many pairs of opposing $A$ and $C$ for the same $\eta$. The model approximates the viscositytemperature curve exactly as well as the Arrhenius model. However, if, e. g., parameter $A$ is used for condition monitoring, changes are artifacts, having no diagnostic value.
Related, but less obvious problems occur if the considered temperature range is too small. E. g., if $B / T$ in the Arrhenius model is close to constant, it behaves similar to $C$ in (8), introducing larger covariance between $A$ and $B$ in case of measurement noise. The German industrial norm DIN 53017 [26] shows three models that are used for approximating the viscosity-temperature characteristics:

$$
\eta=A \exp \frac{B}{\vartheta+C} \quad \ldots \text { Vogel, }
$$

$$
\ln (\eta)=a-b \ln (1-c / T)
$$

$\log \log (v+c)=K_{v}-m \log (T) \quad \ldots$ Ubbelohde-Walther.

Here, $\ln ()$ denotes the natural and $\log ()$ the base-10 logarithm, respectively. Fig. 7 shows 5 different technical fluids, an engine oil 5W30, the hydraulic oil HLP46, the heat carrier fluid Marlotherm SH, the gear oil 80W90 and the viscosity standard fluid S200 from the Cannon instrument company consisting of 1-decene, homopolymer, hydrogenated. The measured temperature characteristics are approximated with the Arrhenius [24], the Vogel [27], the Sturm [28], and the Ubbelohde-Walther [29] model, where for the last model the results were converted to the dynamic viscosity using the values of the known massdensities. The measurement values were recorded within $0^{\circ} \mathrm{C}$ and $80^{\circ} \mathrm{C}$ with a $2{ }^{\circ} \mathrm{C}$ interval. All data points were considered for the determination of the model parameters using least-squares optimization. Table 1 lists the average deviation in mPas for each fluid and model. Among the models, the Vogel model shows the best overall agreement for the considered fluids, but their parameters are difficult to interpret. We propose an alternative representation of the Vogel model

$$
\eta(\vartheta)=\eta_{0} \exp \left(-U_{0} \frac{\left(\vartheta-\vartheta_{0}\right)\left(\vartheta_{0}+C\right)}{C+\vartheta}\right),
$$

where $\eta_{0}$ is the viscosity at reference temperature $\vartheta_{0}$ and $U_{0}$ is the negative temperature coefficient

$$
U_{0}=-\left.\frac{1}{\eta} \frac{d \eta}{d \vartheta}\right|_{\vartheta=9_{0}}
$$

as defined in [26] and shown in Fig. 7b by the tangents at $\vartheta_{0}=40^{\circ} \mathrm{C}$. The correspondence to the $A$ and $B$ parameters of the original Vogel model is given by

$$
\eta_{0}=A \exp \left(\frac{B}{\vartheta_{0}+C}\right) \text { and } U_{0}=\frac{B}{\left(\vartheta_{0}+C\right)^{2}}
$$




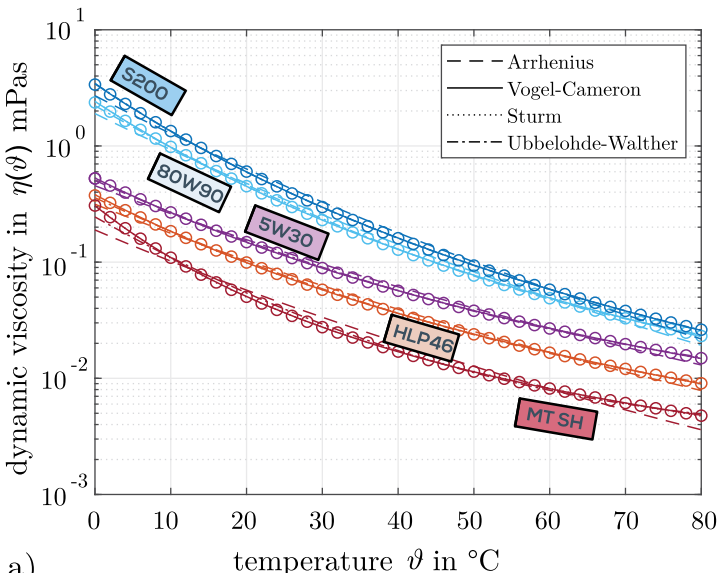

a)

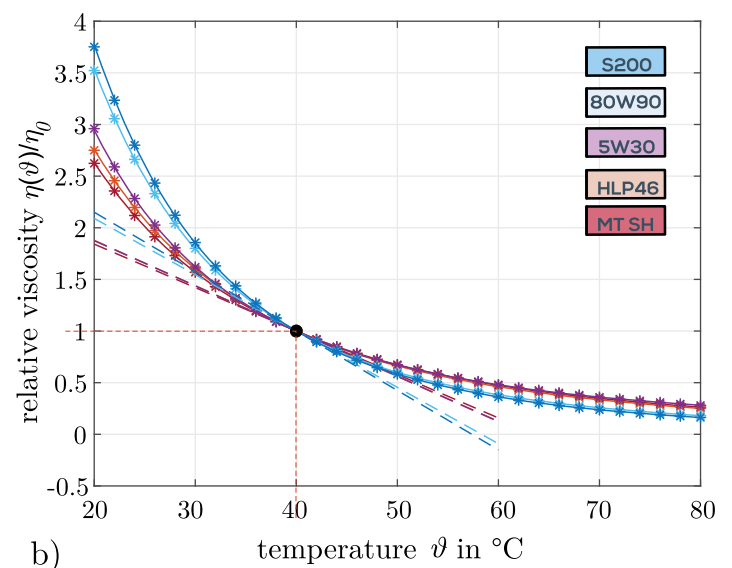

b)

Figure 7: a) Measured viscosities (o) for 5 fluids approximated by different fluid models. b) The negative slope of relative viscosity at $\vartheta_{0}=40^{\circ} \mathrm{C}$ corresponds to $U_{0}$.

Table 1: Average relative deviation in \% between measured dynamic viscosities and the fitting models. The Vogel model fits best for all considered fluids.

\begin{tabular}{lrrrrr}
\hline Fluid & $\eta_{\text {40 }}$ (mPas) & Arrhenius [24] & Vogel [27] & Sturm [28] & Ubbel.-W. [29] \\
\hline 5W30 & 56.64 & 6.25 & 0.31 & 0.45 & 1.29 \\
HLP46 & 36.10 & 6.58 & 0.36 & 0.52 & 1.29 \\
MTSH & 16.98 & 14.79 & 0.32 & 0.49 & 4.45 \\
80W90 & 128.03 & 8.41 & 0.52 & 0.73 & 1.84 \\
S200 & 160.6 & 8.23 & 0.55 & 0.74 & 0.53 \\
\hline
\end{tabular}

with parameter $C$ remaining unchanged. The determination of the $A, B, C$ parameters of the Vogel model employing basic algebraic manipulation using three temperature points is shown in DIN 53017 along with a rule for the spacing of the temperature points depending on the measurement uncertainty of the viscositmeter. In the Appendix, we propose a least-squares regression for the parameters when $N>3$ temperature measurements are available. As will be shown in the results section, the covariance of the $\eta_{0}, U_{0}$, and $C$ parameters is smaller than for $A, B$, and $C$, which make them more reliable monitoring parameters.

\section{Results}

Figure 8 shows the schematic and the photograph of the measurement system attached to a permanently operating oil circuit. The fluidFOX instrument samples the fluid by means of magnetic valved and conditions the fluid sample to the temperature setpoints $20,40,60$, and $80^{\circ} \mathrm{C}$. Figure 9 shows the raw data readings for temperature and viscosity for three exemplary cycles, with a duration of each cycle of approximately 23 minutes. Figure 10a shows the dy- namic viscosity at $40^{\circ} \mathrm{C}$ recorded throughout 6.5 months. On the 8th of December and on the 1st of January, water (approximately 2-5\%) suddenly leaked into the fluid circuit where it formed an emulsion indicated by turbidity visible to the naked eye and accompanied by a temporal increase in viscosity. The first water break-in is shown in b) in more detail where the viscosities for the four temperature setpoints normalized to the respective value before water break-in are depicted. The increase reduced gradually close to the initial value within two days as the water was separated. The temporal spreading of the curves suggests that a change in temperature coefficient has occurred. This is reflected in the calculated negative temperature coefficient $U_{0}$ and in the temperature offset parameter $C$ in Fig. 11.

The benefit of using the transformed parameters for condition monitoring is demonstrated by analyzing how measurement noise affects the estimated parameters $A, B$ and $C$ of the Vogel model and $\eta_{0}, U_{0}$ and $C$ of the proposed transformed Vogel model. The segment of data where only little changes were observed (indicated by the rectangular box in Fig 10a) was used. Figure 12 shows the scatterplots for the relative changes of the estimated parameters for the transformed model (a) and the original Vogel model (b). 


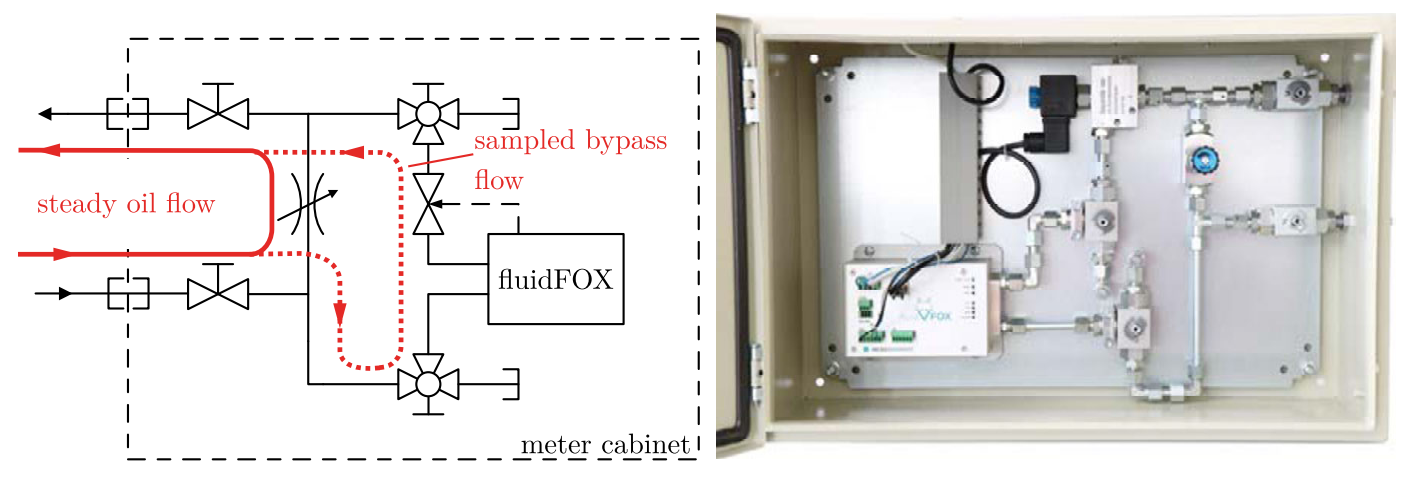

Figure 8: Schematic and image of the condition monitoring setup in the metering cabinet.

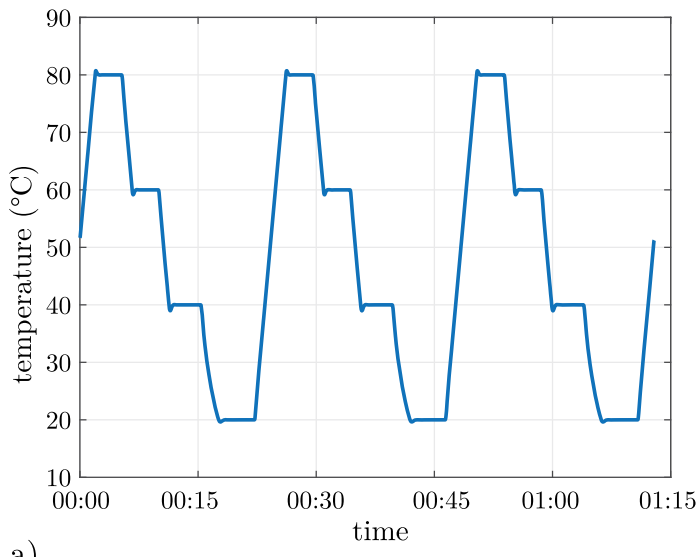

a)

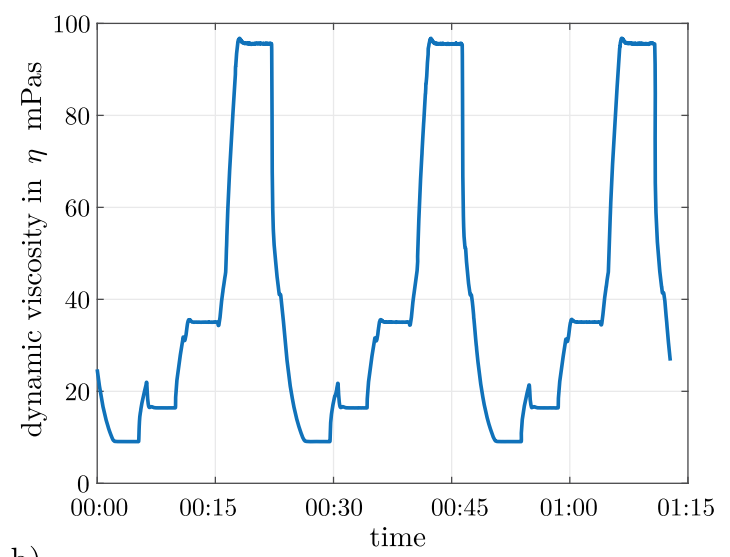

b)

Figure 9: Exemplary temperature cycles (a) and the associated dynamic viscosity (b) measured by the condition monitoring system. The setpoints are $20,40,60$, and $80^{\circ} \mathrm{C}$. One full cycle is obtained within approximately 23 minutes.
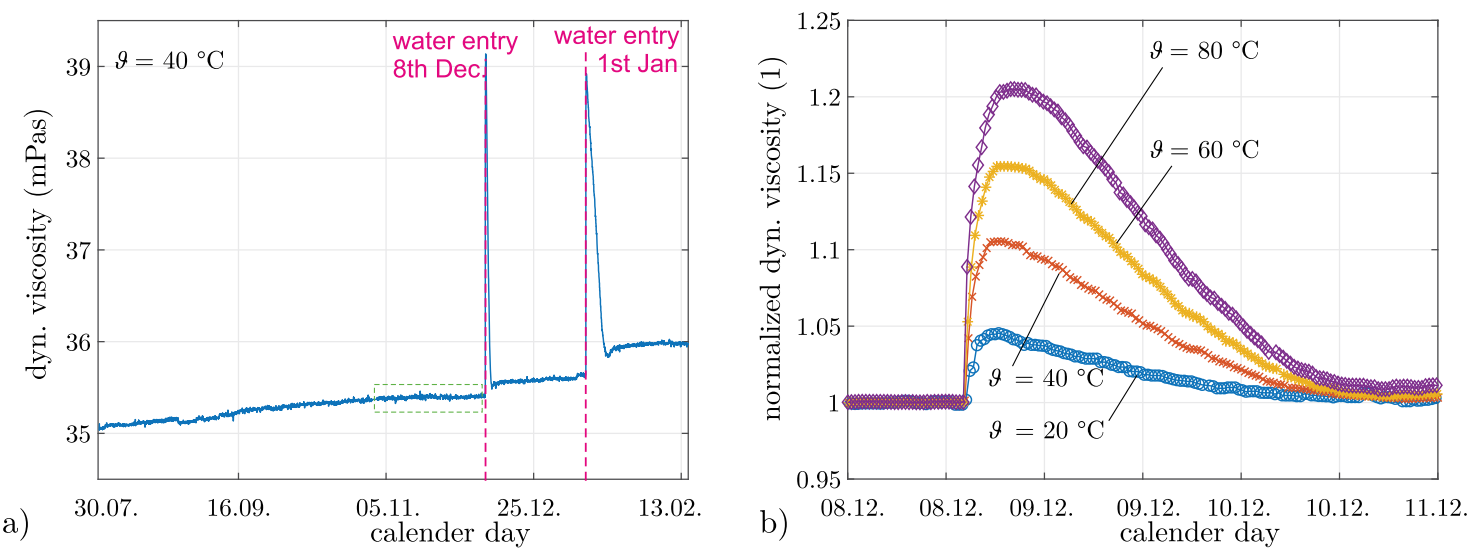

Figure 10: Viscosity changes during the experiment. Sudden water contaminations occurred on the 8th of December and the 1st of January. Temporary increase of viscosity due to water penetration at various temperature setpoints. The values are normalized to the value before water penetration.

Ideally, the axes of the ellipses formed by the points coincide with the main axes as is nearly the case for the transformed model. The thin tilted point ellipses for the $A, B$ and $C$ parameters of the original Vogel model in $\mathrm{b}$ ) reveal clear cross-correlations. The $A$ and $B$ parameters counteract, making them less suitable for condition monitoring as they are noisier or their changes may even be artificial. It is pointed out, that these correlations can also be calculated 

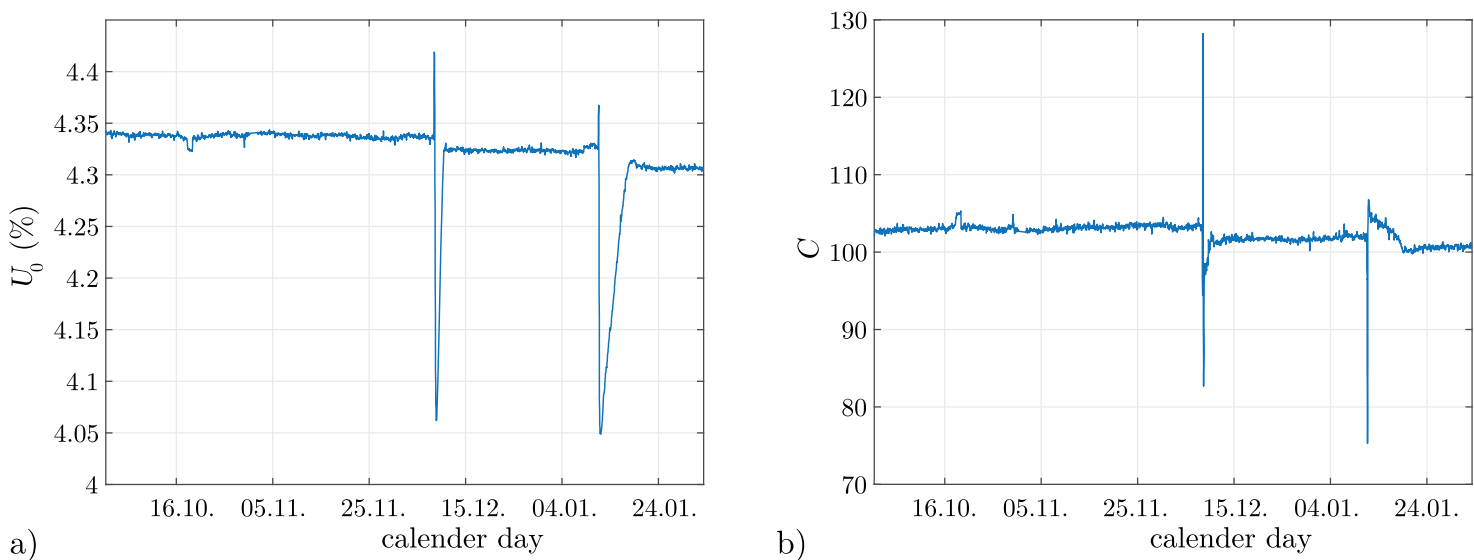

Figure 11: Estimated temperature coefficient $U_{0}(\mathrm{a})$ and temperature offset $C$ (b) of the proposed transformed Vogel model.
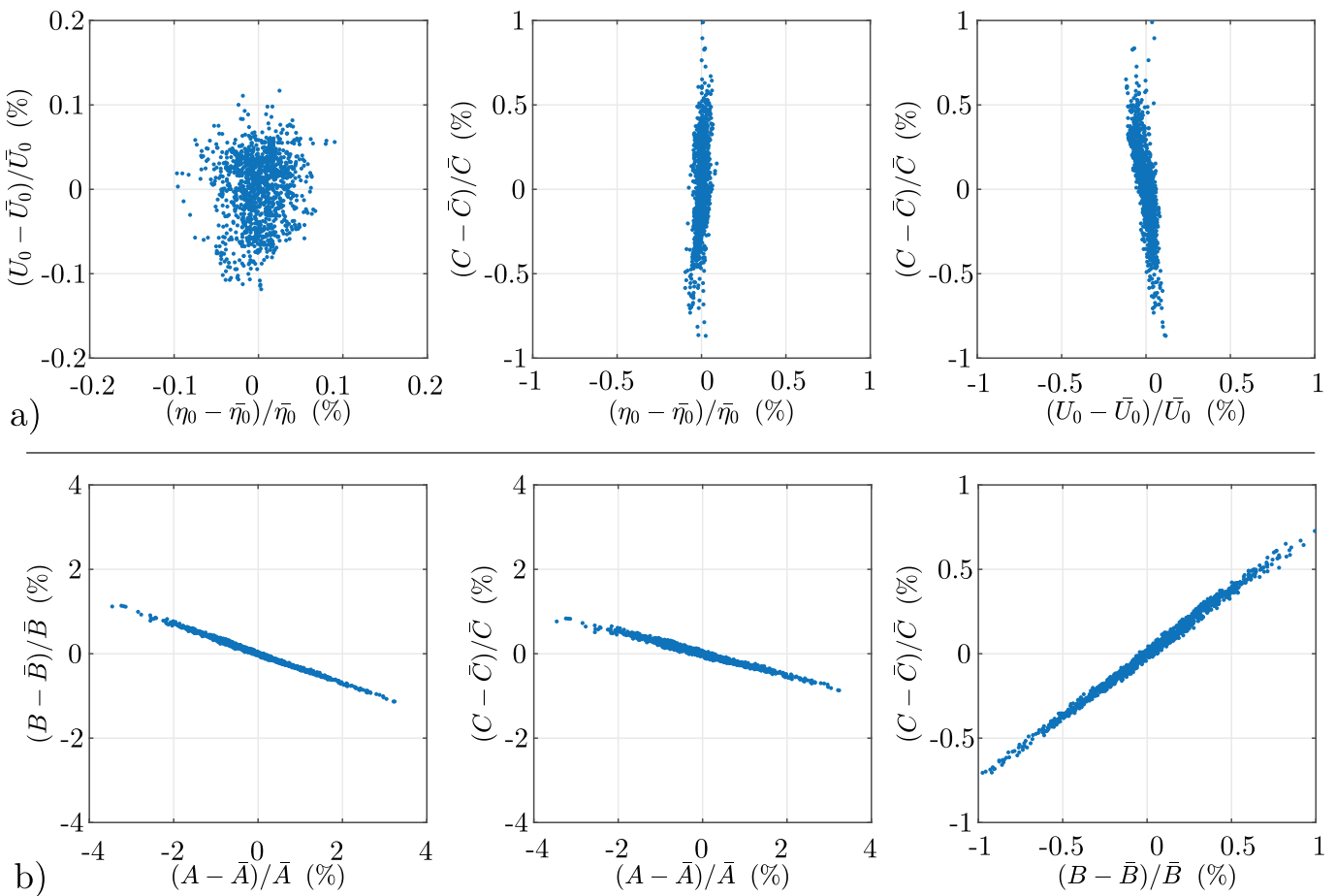

Figure 12: The upper row of figures (a) shows the scatter plots of the estimated relative parameter changes of the transformed Vogel model. Covariances are small indicating that these monitoring parameters are stable under measurement noise. The lower row of figures (b) shows the scatter plots for the parameters of the Vogel model. Left-leaning error ellipses indicate counteracting parameters in presence of measurement noise.

from the models directly by means of covariance matrices employing fundamental estimation theory [30, 31], which however is not further considered here.

\section{Conclusions}

An extended model for the fluid-to-resonance relation has been demonstrated, enabling increased accuracy and measurement range for a recently introduced novel resonant sensor system for oil condition monitoring. These benefits were substantiated by comparison to alternative models. Furthermore, extended fluid monitoring capabilities determining the temperature characteristic of the fluid viscosity were investigated. We identified the Vogel relation as a suitable viscosity-temperature model for a group of technically relevant fluids requiring a minimum of parameters, which however have no clear physical mean- 
ing as is desired for condition monitoring purposes. The proposed transformation not only makes an interpretation clearer but reduces also the covariance of the parameters. The temperature parameters were shown for a long-term experiment in a hydraulic rail where sudden water leakage faults could be clearly and immediately be resolved by the system. Such transient events may not be recognized by coarse bottle sampling.

Funding: This research was partly supported by Linz Center of Mechatronics (LCM) in the framework of the Austrian COMET-K2 programme.

\section{Appendix}

We propose a transformation of (9) introducing two parameters $\alpha$ and $\beta$

$$
\frac{\vartheta-\vartheta_{1}}{\ln \left(\eta_{1}\right)-\ln (\eta)}=\alpha+\left(\vartheta-\vartheta_{1}\right) \beta
$$

Here $\vartheta_{1}$ is an arbitrary temperature and $\eta_{1}$ the associated viscosity. Given $N$ measurements at the respective temperatures $\vartheta_{[1]} \ldots \vartheta_{[N]}$ and the associated viscosities $\eta_{[1]} \ldots \eta_{[N]}$, a linear equation system can be established

$$
\underbrace{\left[\begin{array}{c}
\left(\vartheta_{[1]}-\vartheta_{1}\right) /\left(\ln \left(\eta_{1}\right)-\ln \left(\eta_{[1]}\right)\right) \\
\left(\vartheta_{[2]}-\vartheta_{1}\right) /\left(\ln \left(\eta_{1}\right)-\ln \left(\eta_{[2]}\right)\right) \\
\vdots \\
\left(\vartheta_{[N]}-\vartheta_{1}\right) /\left(\ln \left(\eta_{1}\right)-\ln \left(\eta_{[N]}\right)\right)
\end{array}\right]}_{\boldsymbol{x}}=\underbrace{\left[\begin{array}{cc}
1 & \vartheta_{[1]}-\vartheta_{1} \\
1 & \vartheta_{[2]}-\vartheta_{1} \\
\vdots & \vdots \\
1 & \vartheta_{[N]}-\vartheta_{1}
\end{array}\right]}_{\boldsymbol{H}} \cdot \underbrace{\left[\begin{array}{l}
\alpha \\
\beta
\end{array}\right]}_{\boldsymbol{\theta}} .
$$

If $\vartheta_{0}$ is within the set of measured temperatures, the respective line can be canceled from the equation system to avoid $1 / 0$ division. (It can be shown that the limit of the respective $x$-value converges to $\alpha$.) The parameter vector $\boldsymbol{\theta}$ is estimated by least-squares regression, and the estimates are indicated by the hat symbol

$$
\hat{\boldsymbol{\theta}}=\left(\boldsymbol{H}^{\mathrm{T}} \cdot \boldsymbol{H}\right)^{-1} \cdot \boldsymbol{H}^{\mathrm{T}} \cdot \boldsymbol{x}
$$

Finally, estimates for $B$ and $C$ are obtained by applying the manipulations

$$
\hat{B}=\hat{\alpha} / \hat{\beta}^{2} \text { and } \hat{C}=\hat{\alpha} / \hat{\beta}-\vartheta_{1}
$$

By comparing the result to (14), it is found that $1 / \alpha$ equals the temperature coefficient $U$ at $\vartheta_{1}$, such that if $\vartheta_{1}=\vartheta_{0}$ is chosen, $U_{0}$ is directly obtained by the regression. A leastsquares regression for $A$ is e. g., obtained by using (16) with $\boldsymbol{x}=\boldsymbol{\eta}$ and

$$
\boldsymbol{H}=\left[\begin{array}{c}
\exp \left(\hat{B} /\left(\vartheta_{[1]}+\hat{C}\right)\right) \\
\vdots \\
\exp \left(\hat{B} /\left(\vartheta_{[N]}+\hat{C}\right)\right)
\end{array}\right]
$$

\section{References}

1. Thomas Voglhuber-Brunnmaier, Alexander O Niedermayer, Friedrich Feichtinger, and Bernhard Jakoby. E6.3 an advanced multi-parameter condition monitoring system for lubricants and hydraulic fluids. In: SMSI 2020-Measurement Science, pages 313-314, 2020.

2. Thomas Voglhuber-Brunnmaier, Alexander O Niedermayer, Friedrich Feichtinger, and Bernhard Jakoby. C6.3 advanced fluid models for multi-parameter condition monitoring systems for lubricants and hydraulic fluids. In: SMSI 2021-Measurement Science, pages 216-217, 2021.

3. Ramesh Gulati and Ricky Smith. Maintenance and reliability best practices. Industrial Press Inc., 2009.

4. Marcus Bengtsson and Gunnar Lundström. On the importance of combining "the new" with "the old"-one important prerequisite for maintenance in industry 4.0. Procedia Manufacturing, 25:118-125, 2018.

5. Accelic Connected Reliability. Potential-failure ( $p-f)$ curve faq. online, accessed: 21/8/17.

6. R Keith Mobley. An introduction to predictive maintenance. Elsevier, 2002.

7. Micro Resonant Technologies GmbH. Homepage. http://www. micro-resonant.at/cms. Accessed: 2021-09-30.

8. Thomas Voglhuber-Brunnmaier, Alexander O Niedermayer, Friedrich Feichtinger, Erwin K Reichel, and Bernhard Jakoby. A multi-parameter physical fluid sensor system for industrial and automotive applications. tm-Technisches Messen, 87(3):189-200, 2020.

9. Alexander O Niedermayer, Thomas Voglhuber-Brunnmaier, Friedrich Feichtinger, Martin Heinisch, and Bernhard Jakoby. Online condition monitoring of lubricating oil based on resonant measurement of fluid properties. In Sensors and Measuring Systems; 19th ITG/GMA-Symposium, pages 1-4. VDE, 2018.

10. Alexander $O$ Niedermayer, Thomas Voglhuber-Brunnmaier, Martin Heinisch, and Bernhard Jakoby. A2.4 accurate determination of viscosity and mass density of fluids using a piezoelectric tuning fork resonant sensor. In: Proceedings SENSOR 2015, pages 66-71, 2015.

11. Thomas Voglhuber-Brunnmaier, Alexander 0 Niedermayer, Friedrich Feichtinger, and Bernhard Jakoby. Fluid sensing using quartz tuning forks: Measurement technology and applications. Sensors, 19(10):2336, 2019.

12. Theo Mang and Wilfried Dresel. Lubricants and lubrication. John Wiley \& Sons, 2007.

13. Lew D Landau and Evgeny M Lifshitz. Theoretical physics, vol. 6, Fluid mechanics (p. 89). Pergamon, London, 1987. 
14. Ernest $O$ Tuck. Calculation of unsteady flows due to small motions of cylinders in a viscous fluid. Journal of Engineering Mathematics, 3(1):29-44, 1969.

15. Martin Heinisch, Thomas Voglhuber-Brunnmaier, Erwin K Reichel, Isabelle Dufour, and Bernhard Jakoby. Reduced order models for resonant viscosity and mass density sensors. Sensors and Actuators A: Physical, 220:76-84, 2014.

16. Mohamed Youssry, Naser Belmiloud, Benjamin Caillard, Cédric Ayela, Claude Pellet, and Isabelle Dufour. A straightforward determination of fluid viscosity and density using microcantilevers: from experimental data to analytical expressions. Sensors and Actuators A: Physical, 172(1):40-46, 2011.

17. Javier Toledo, Thomás Manzaneque, Victor Ruiz-Díez, Martin Kucera, Georg Pfusterschmied, Elisabeth Wistrela, Ulrich Schmid, and Jose L Sánchez-Rojas. Piezoelectric resonators and oscillator circuit based on higher-order out-of-plane modes for density-viscosity measurements of liquids. Journal of Micromechanics and Microengineering, 26(8):084012, 2016.

18. Mi Zhang, Dehua Chen, Xiao He, and Xiuming Wang. A hydrodynamic model for measuring fluid density and viscosity by using quartz tuning forks. Sensors, 20(1):198, 2020.

19. Thomas Voglhuber-Brunnmaier and Bernhard Jakoby. Higher-order models for resonant viscosity and mass-density sensors. Sensors, 20(15):4279, 2020.

20. Oliver Aberth. Iteration methods for finding all zeros of a polynomial simultaneously. Mathematics of Computation, 27(122):339-344, 1973.

21. Dario Andrea Bini. Numerical computation of polynomial zeros by means of Aberth's method. Numerical Algorithms, 13(2):179-200, 1996.

22. A Wilmer Duff. Empirical formulæ for viscosity as a function of temperature. Phys. Rev. (Series I), 4:404-410, Mar 1897.

23. Jean LM Poiseuille. Experimentelle Untersuchungen über die Bewegung der Flüssigkeiten in Röhren von sehr kleinen Durchmessern. Annalen der Physik, 134(3):424-448, 1843.

24. Svante Arrhenius. Über die Reaktionsgeschwindigkeit bei der Inversion von Rohrzucker durch Säuren. Zeitschrift für physikalische Chemie, 4(1):226-248, 1889.

25. Martin Heinisch. Mechanical resonators for liquid viscosity and mass density sensing. $\mathrm{PhD}$ thesis, Université de Bordeaux, 2015.

26. DIN e. V. (Hrsg.) DIN 53017: 1993, Bestimmung des Temperaturkoeffizienten der Viskosität von Flüssigkeiten, Beuth-Verlag, Berlin, 1993, November 1993.

27. H Vogel. Das Temperaturabhängigkeitsgesetz der Viskosität von Flüssigkeiten. Phys. Zeitschr, 22:645-646, 1921.

28. Karl G Sturm. Thermische Ausdehnung und Glasübergang als Bestimmungsgrößen der Viskosität von Flüssigkeiten. Rheologica Acta, 20(1):59-63, 1981.

29. Leo Ubbelohde. Zur Viskosimetrie mit Umwandlungs-und Rechentabellen. Hirzel, 1965.

30. Steven M Kay. Fundamentals of statistical signal processing: estimation theory. Prentice-Hall, Inc., 1993.

31. Thomas Voglhuber-Brunnmaier, Alexander O Niedermayer, Martin Heinisch, Ali Abdallah, Erwin K Reichel, Bernhard Jakoby, Veronika Putz, and Roman Beigelbeck. Modeling-free evaluation of resonant liquid sensors for measuring viscosity and density. In 2015 9th International Conference on Sensing Technology (ICST), pages 300-305. IEEE, 2015.

\section{Bionotes}

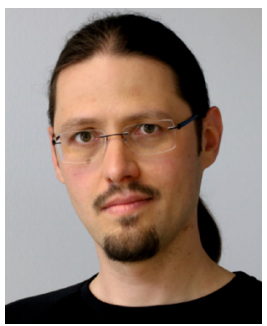

Thomas Voglhuber-Brunnmaier Institute for Microelectronics and Microsensors, Johannes Kepler University, Linz, Austria thomas.voglhuber-brunnmaier@jku.at

Thomas Voglhuber-Brunnmaier received the Dipl.-Ing. (M. Sc.) degree in Mechatronics from Johannes Kepler University (JKU), Linz, Austria, in 2007 and the Ph. D. in 2013 at the Institute for Microelectronics and Microsensors (IME) at JKU. From 2013 to 2016 he was working with the Center for Integrated Sensor Systems at the Danube University Krems and from 2017 to 2019 at the Linz Institute of Technology (LIT). He is currently working as a senior researcher at IME. His fields of interest are the modeling of microsensors for fluid sensing, material characterization and measurement science.

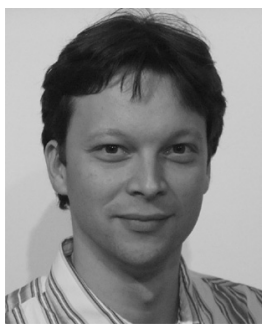

Alexander 0. Niedermayer

Micro Resonant Technologies GmbH, Linz, Austria

a.niedermayer@micro-resonant.com

Alexander O. Niedermayer received the Dipl.-Ing. (M. Sc.) degree in Mechatronics in 2007 and the Ph. D. degree in 2013 from Johannes Kepler University, Linz, Austria, where he was working on interface circuits for resonant sensors and optimized resonance estimation methods. He is founder of Micro Resonant Technologies company established in 2014.

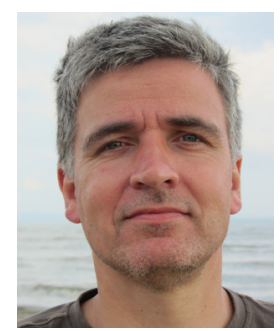

\section{Bernhard Jakoby}

Institute for Microelectronics and

Microsensors, Johannes Kepler University, Linz, Austria

bernhard.jakoby@jku.at

Bernhard Jakoby obtained his Dipl.-Ing. (M. Sc.) in Communication Engineering and his doctoral (Ph. D.) degree in electrical engineering from the Vienna University of Technology (VUT), Austria, in 1991 and 1994, respectively. In 2001 he obtained avenia legendi for Theoretical Electrical Engineering from the VUT. From 1991 to 1994 he worked as a Research Assistant at the Institute of General Electrical Engineering and Electronics of the VUT. Subsequently he stayed as an Erwin Schrödinger Fellow at the University of Ghent, Belgium, performing research on the electrodynamics of complex media. 
From 1996 to 1999 he held the position of a Research Associate and later Assistant Professor at the Delft University of Technology, The Netherlands, working in the field of microacoustic sensors. From 1999 to 2001 he was with the Automotive Electronics Division of the Robert Bosch GmbH, Germany, where he conducted development projects in the field of automotive liquid sensors. In 2001 he joined the newly formed Industrial Sensor Systems group of the VUT as an Associate Professor. In 2005 he was appointed Full Professor of Microelectronics at the Johannes Kepler University Linz, Austria. He is currently working in the field of fluidic sensors, integrated photonic sensors, and monitoring systems. 